\title{
A random model for immune response to virus in fluctuating environments
}

\author{
Yusuke Asai, Tomás Caraballo, Xiaoying Han and Peter E. Kloeden
}

\begin{abstract}
In this work we study a model for virus dynamics with a random immune response and a random production rate of susceptible cells from cell proliferation. In traditional models for virus dynamics, the rate at which the viruses are cleared by the immune system is constant, and the rate at which susceptible cells are provided is constant or a function depending on the population of all cells. However, the human body in general is never stationary, and thus these rates can barely be constant. Here we assume that the human body is a random environment and models the rates by random processes, which result in a system of random differential equations. We then analyze the long term behavior of the random system, in particular the existence and geometric structure of the random attractor, by using the theory of random dynamical systems. Numerical simulations are provided to illustrate the theoretical result.
\end{abstract}

Yusuke Asai

Institut für Biostatistik und mathematische Modellierung, Klinikum und Fachbereich Medizin, Goethe-Universität, D-60590 Frankfurt am Main, Germany. e-mail: asai@med.uni-frankfurt.de

Tomás Caraballo

Dpto. Ecuaciones Diferenciales y Análisis Numérico, Universidad de Sevilla, Apdo. de Correos 1160, 41080-Sevilla, Spain.e-mail: caraball@us.es

Xiaoying Han

221 Parker Hall, Department of Mathematics and Statistics, Auburn University, Auburn, AL 36849

USA.e-mail: xzh0003@auburn.edu

Peter E. Kloeden

School of Mathematics and Statistics, Huazhong University of Science \& Technology, Wuhan 430074, China, and Felix-Klein-Zentrum für Mathematik, TU Kaiserslautern, D-67663 Kaiserslautern, Germany. e-mail: kloeden@mathematik.uni-kl.de 


\section{Introduction}

Basic models for virus dynamics were introduced in the classic text by May and Nowak [12]. The assumption for simplest models is that the body is modeled as a "well stirred" chemostat containing the virus and two kinds of cells, uninfected but susceptible cells and cells infected by virus. In a chemostat, microorganisms grow by feeding on nutrients in the culture vessel, and are flushed out to the collecting vessel. Similarly in the human body, the virus grows from dead infected cells and is cleared by the immune system. Modeling chemostats by systems of nonautonomous or random differential equations is fully justified (see, e.g., [6, 7]), as the environment for a chemostat usually varies in time (either deterministically or randomly). Using the argument that the human body also varies in time, we will model the virus dynamics by a system of random differential equations in this work.

Denote by $v$ the population size of free virus, $x$ the population size of uninfected cells (food for virus), and $y$ the population size of infected cells. First uninfected cells are produced by cell proliferation at a constant rate $\Lambda$, live for an average lifetime and die at an average death rate $\gamma_{1}$. Second, virus infects susceptible cells to produce infected cells, with an "efficiency", $\beta$. Since cells are infected by contact with viruses, the infection can be modeled as a simple mass action reaction

$$
x+v \stackrel{\beta}{\longrightarrow} y .
$$

Third, infected cells die at an average rate $\gamma_{2}$ and release new viruses at a rate $\kappa$. At the same time these viruses are cleared by the immune system at a rate $\alpha$. Then we arrive at the basic model of virus dynamics:

$$
\begin{aligned}
& \frac{\mathrm{d} x(t)}{\mathrm{d} t}=\Lambda-\gamma_{1} x-\beta x v, \\
& \frac{\mathrm{d} y(t)}{\mathrm{d} t}=\beta x v-\gamma_{2} y, \\
& \frac{\mathrm{d} v(t)}{\mathrm{d} t}=\kappa y-\alpha v .
\end{aligned}
$$

The ordinary differential equation system (1) - (3) can be used to describe the dynamics of various type of virus, healthy and infected cells, but with limitations. First, the model assumes that the contribution of the immune response (to the death of infected cells or free virus and to reducing the rate of infection of new cells) is constant over time. Second, the dynamics of the susceptible cell population assumes a constant production rate from a pool of precursors. These assumptions may be justified for stationary environments, within a short term of time span. However, in the long term, the human body is never a stationary environment - it varies over time in principle and hence system (1) - (3) is not adequate to explain the real dynamic of virus and the immune response. 
In this work, we will assume that the human body is a random environment, that varies randomly with respect to time. Due to this random variation, the contribution of the immune response and the production rate of susceptible cells from cell proliferation will also fluctuate randomly with respect to time. More precisely, we assume that parameters $\Lambda$ and $\alpha$ are perturbed by real noise, i.e., $\Lambda=\Lambda\left(\theta_{t} \omega\right)$ and $\alpha=\alpha\left(\theta_{t} \omega\right)$ are continuous and essentially bounded:

$$
\begin{array}{ccc}
\Lambda\left(\theta_{t} \omega\right) \in \lambda \cdot\left[1-\delta_{1}, 1+\delta_{1}\right], & \lambda>0, & 0<\delta_{1}<1, \\
\alpha\left(\theta_{t} \omega\right) \in a \cdot\left[1-\delta_{2}, 1+\delta_{2}\right], & a>0, & 0<\delta_{2}<1 .
\end{array}
$$

Then system (1) - (3) becomes

$$
\begin{aligned}
& \frac{\mathrm{d} x(t, \omega)}{\mathrm{d} t}=\Lambda\left(\theta_{t} \omega\right)-\gamma_{1} x-\beta x v \\
& \frac{\mathrm{d} y(t, \omega)}{\mathrm{d} t}=\beta x v-\gamma_{2} y \\
& \frac{\mathrm{d} v(t, \omega)}{\mathrm{d} t}=\kappa y-\alpha\left(\theta_{t} \omega\right) v
\end{aligned}
$$

where $\gamma_{1}, \gamma_{2}, \beta, \kappa$ are positive constants, and $\Lambda\left(\theta_{t} \omega\right)$ and $\alpha\left(\theta_{t} \omega\right)$ are defined as in (4) and (5), respectively.

Bounded noise can be modeled in various ways. For example in [2], given a stochastic process $Z_{t}$ such as an Ornstein-Uhlenbeck (OU) process, the stochastic process

$$
\zeta\left(Z_{t}\right):=\zeta_{0}\left(1-2 \varepsilon \frac{Z_{t}}{1+Z_{t}^{2}}\right),
$$

where $\zeta_{0}$ and $\varepsilon$ are positive constants with $\varepsilon \in(0,1)$, takes values in the interval $\zeta_{0}[1-\varepsilon, 1+\varepsilon]$ and tends to peak around $\zeta_{0}(1 \pm \varepsilon)$. It is thus suitable for a noisy switching scenario. In another example, the stochastic process

$$
\eta\left(Z_{t}\right):=\eta_{0}\left(1-\frac{2 \varepsilon}{\pi} \arctan Z_{t}\right),
$$

where $\eta_{0}$ and $\varepsilon$ are positive constants with $\varepsilon \in(0,1)$ takes values in the interval $\eta_{0}[1-\varepsilon, 1+\varepsilon]$ and is centered on $\eta_{0}$. In the theory of random dynamical systems the driving noise process $Z_{t}(\omega)$ is replaced by a canonical driving system $\theta_{t} \omega$. This simplification allows a better understanding of the path-wise approach to model noise: a system influenced by stochastic processes for each single realization $\omega$ can be interpreted as wandering along a path $\theta_{t} \omega$ in $\Omega$ and thus may provide additional statistical information to the modeler.

In the paper we will study the properties of solutions to (6) - (8). In particular, we are interested in the long term behavior of solutions to (6) - (8), characterized by a global random attractor. The rest of the paper is organized as follows. In Section 2 we provide preliminaries on the theory of random dynamical systems. In Section 
3 we prove the existence and uniqueness of a positive bounded solution to (6) (8), and show that the solution generates a random dynamical system. In section 4 we prove the existence and uniqueness of a global random attractor to the random dynamical system generated by the solution to $(6)-(8)$, and also investigate the conditions under which the global random attractor consists of a singleton axial solution (endemic), or nontrivial component sets (pandemic). Numerical simulations are provided in Section 6, to illustrate the conditions for the endemic and pandemic of system (6) - (8).

\section{Preliminaries on random dynamical systems}

In this section we first present some concepts (from [1]) related to general random dynamical systems (RDSs) and random attractors that we require in the sequel. Our situation is, in fact, somewhat simpler, but to facilitate the reader's access to the literature we give more general definitions here.

Let $\left(X,\|\cdot\|_{X}\right)$ be a separable Banach space and let $(\Omega, \mathscr{F}, \mathscr{P})$ be a probability space where $\mathscr{F}$ is the $\sigma$-algebra of measurable subsets of $\Omega$ (called "events") and $\mathscr{P}$ is the probability measure. To connect the state $\omega$ in the probability space $\Omega$ at time 0 with its state after a time of $t$ elapses, we define a flow $\theta=\left\{\theta_{t}\right\}_{t \in \mathbb{R}}$ on $\Omega$ with each $\theta_{t}$ being a mapping $\theta_{t}: \Omega \rightarrow \Omega$ that satisfies

(1) $\theta_{0}=\operatorname{Id}_{\Omega}$,

(2) $\theta_{s} \circ \theta_{t}=\theta_{s+t}$ for all $s, t \in \mathbb{R}$,

(3)the mapping $(t, \omega) \mapsto \theta_{t} \omega$ is measurable and

(4)the probability measure $\mathscr{P}$ is preserved by $\theta_{t}$, i.e. $\theta_{t} \mathscr{P}=\mathscr{P}$.

This set-up establishes a time-dependent family $\theta$ that tracks the noise, and $(\Omega, \mathscr{F}, \mathscr{P}, \theta)$ is called a metric dynamical system [1].

Definition 1. A stochastic process $\{S(t, \omega)\}_{t \geq 0, \omega \in \Omega}$ is said to be a continuous $\operatorname{RDS}$ over $\left(\Omega, \mathscr{F}, \mathbb{P},\left(\theta_{t}\right)_{t \in \mathbb{R}}\right)$ with state space $X$ if $S:[0,+\infty) \times \Omega \times X \rightarrow X$ is $(\mathscr{B}[0,+\infty) \times \mathscr{F} \times \mathscr{B}(X), \mathscr{B}(X))$ - measurable, and for each $\omega \in \Omega$,

(1)the mapping $S(t, \omega): X \rightarrow X, x \mapsto S(t, \omega) x$ is continuous for every $t \geq 0$;

(2) $S(0, \omega)$ is the identity operator on $X$;

(3)(cocycle property) $S(t+s, \omega)=S\left(t, \theta_{s} \omega\right) S(s, \omega)$ for all $s, t \geq 0$.

Definition 2. (1)A set-valued mapping $B: \omega \rightarrow 2^{X} \backslash \emptyset$ is said to be a random set if the mapping $\omega \mapsto \operatorname{dist}_{X}(x, B(\omega))$ is measurable for any $x \in X$.

(2)A random set $B(\omega)$ is said to be bounded if $B(\omega)$ is bounded for a.e. $\omega \in \Omega$; a random set $B(\omega)$ is said to be compact if $B(\omega)$ is compact for a.e. $\omega \in \Omega$; a random set is said to be closed if $B(\omega)$ is closed for a.e. $\omega \in \Omega$. 
(3)A bounded random set $B(\omega) \subset X$ is said to be tempered with respect to $\left(\theta_{t}\right)_{t \in \mathbb{R}}$ if for a.e. $\omega \in \Omega$,

$$
\lim _{t \rightarrow \infty} e^{-\beta t} \sup _{x \in B\left(\theta_{-t} \omega\right)}\|x\|_{X}=0, \quad \text { for all } \beta>0
$$

a random variable $\omega \mapsto r(\omega) \in \mathbb{R}$ is said to be tempered with respect to $\left(\theta_{t}\right)_{t \in \mathbb{R}}$ if for a.e. $\omega \in \Omega$,

$$
\lim _{t \rightarrow \infty} e^{-\beta t} \sup _{t \in \mathbb{R}}\left|r\left(\theta_{-t} \omega\right)\right|=0, \quad \text { for all } \beta>0 .
$$

In what follows we use $\mathscr{D}(X)$ to denote the set of all tempered random sets of $X$.

Definition 3. A random set $K(\omega) \subset X$ is called a random absorbing set in $\mathscr{D}(X)$ if for any $B \in \mathscr{D}(X)$ and a.e. $\omega \in \Omega$, there exists $T_{B}(\omega)>0$ such that

$$
S\left(t, \theta_{-t} \omega\right) B\left(\theta_{-t} \omega\right) \subset K(\omega), \quad \forall t \geq T_{B}(\omega) .
$$

Definition 4. Let $\{S(t, \omega)\}_{t \geq 0, \omega \in \Omega}$ be an $\operatorname{RDS}$ over $\left(\Omega, \mathscr{F}, \mathbb{P},\left(\theta_{t}\right)_{t \in \mathbb{R}}\right)$ with state space $X$ and let $\mathscr{A}(\omega)(\subset X)$ be a random set. Then $\mathscr{A}(\omega)$ is called a global random $\mathscr{D}$ attractor (or pullback $\mathscr{D}$ attractor) for $\{S(t, \omega)\}_{t \geq 0, \omega \in \Omega}$ if $\omega \mapsto \mathscr{A}(\omega)$ satisfies

(1)(random compactness) $\mathscr{A}(\omega)$ is a compact set of $X$ for a.e. $\omega \in \Omega$;

(2)(invariance) for a.e. $\omega \in \Omega$ and all $t \geq 0$, it holds

$$
S(t, \omega) \mathscr{A}(\omega)=\mathscr{A}\left(\theta_{t} \omega\right)
$$

(3)(attracting property) for any $B \in \mathscr{D}(X)$ and a.e. $\omega \in \Omega$,

$$
\lim _{t \rightarrow \infty} \operatorname{dist}_{X}\left(S\left(t, \theta_{-t} \omega\right) B\left(\theta_{-t} \omega\right), \mathscr{A}(\omega)\right)=0,
$$

where

$$
\operatorname{dist}_{X}(G, H)=\sup _{g \in G} \inf _{h \in H}\|g-h\|_{X}
$$

is the Hausdorff semi-metric for $G, H \subseteq X$.

Proposition 1. $[5,9,10]$ Let $B \in \mathscr{D}(X)$ be an absorbing set for the continuous random dynamical system $\{S(t, \omega)\}_{t \geq 0, \omega \in \Omega}$ which is closed and satisfies the asymptotic compactness condition for a.e. $\omega \in \Omega$, i.e., each sequence $x_{n} \in S\left(t_{n}, \theta_{-t_{n}}, B\left(\theta_{-t_{n}} \omega\right)\right)$ has a convergent subsequence in $X$ when $t_{n} \rightarrow \infty$. Then the cocycle $S$ has a unique global random attractor with component subsets

$$
\mathscr{A}(\omega)=\bigcap_{\tau \geq t_{B}(\omega) t \geq \tau} \overline{\bigcup_{t \geq \tau} S\left(t, \theta_{-t} \omega\right) B\left(\theta_{-t} \omega\right)} .
$$

If the pullback absorbing set is positively invariant, i.e., $S(t, \omega) B(\omega) \subset B\left(\theta_{t} \omega\right)$ for all $t \geq 0$, then 


$$
\mathscr{A}(\omega)=\bigcap_{t \geq 0} S\left(t, \theta_{-t} \omega\right) B\left(\theta_{-t} \omega\right)
$$

For state space $X=\mathbb{R}^{d}$ as in this paper, the asymptotic compactness follows trivially. Note that the random attractor is pathwise attracting in the pullback sense, but need not be pathwise attracting in the forward sense, although it is forward attracting in probability, due to some possible large deviations, see e.g., Arnold [1].

When the cocycle mapping is strictly uniformly contracting $[8,11]$, i.e., there exists $K>0$ such that

$$
\left\|S(t, \omega) x_{0}-S(t, \omega) y_{0}\right\|_{X} \leq e^{-K t}\left\|x_{0}-y_{0}\right\|_{X}
$$

for all $t \geq 0, \omega \in \Omega$ and $x_{0}, y_{0} \in X$, then the random attractor consists of singleton subsets $\mathscr{A}(\omega)=\{A(\omega)\}$. It is thus essentially a single stochastic process with sample paths $A\left(\theta_{t} \omega\right)$ for all $t \in \mathbb{R}$. The proof uses a Cauchy sequence rather than compactness argument. In this case the random attractor is pathwise attracting in both the pullback and forward senses.

\section{Properties of solutions}

In this section we will prove the existence, uniqueness and boundedness of positive solutions to $(6)-(8)$. In addition we prove that the solution generates a random dynamical system. Denote by

$$
\mathbb{R}_{+}^{3}=\left\{(x, y, v) \in \mathbb{R}^{3}: x \geq 0, y \geq 0, v \geq 0\right\},
$$

and for simplicity we write $\mathbf{u}(t, \omega)=(x(t, \omega), y(t, \omega), v(t, \omega))^{T}$.

Theorem 1. For any $\omega \in \Omega, t_{0} \in \mathbb{R}$ and initial data $\boldsymbol{u}_{0}=\left(x\left(t_{0}\right), y\left(t_{0}\right), v\left(t_{0}\right)\right)^{T} \in$ $\mathbb{R}_{+}^{3}$, system (6) - (8) has a unique non-negative bounded solution $\boldsymbol{u}\left(\cdot ; t_{0}, \omega, \boldsymbol{u}_{0}\right) \in$ $\mathscr{C}\left(\left[t_{0}, \infty\right), \mathbb{R}_{+}^{3}\right)$, with $\boldsymbol{u}\left(t_{0} ; t_{0}, \omega, \boldsymbol{u}_{0}\right)=\boldsymbol{u}_{0}$. Moreover, the solution generates a random dynamical system $\varphi(t, \omega)(\cdot)$ defined as

$$
\varphi(t, \omega) \boldsymbol{u}_{0}=\boldsymbol{u}\left(t ; 0, \omega, \boldsymbol{u}_{0}\right), \quad \forall t \geq 0, \boldsymbol{u}_{0} \in \mathbb{R}_{+}^{3}, \omega \in \Omega .
$$

Proof. Write

$$
L\left(\theta_{t} \omega\right)=\left(\begin{array}{ccc}
-\gamma_{1} & 0 & 0 \\
0 & -\gamma_{2} & 0 \\
0 & \kappa & -\alpha\left(\theta_{t} \omega\right)
\end{array}\right) \quad \text { and } \quad f\left(\theta_{t} \omega, \mathbf{u}\right)=\left(\begin{array}{c}
\Lambda\left(\theta_{t} \omega\right)-\beta x v \\
\beta x v \\
0
\end{array}\right),
$$

then equations (6) - (8) become

$$
\frac{\mathrm{d} u(t, \omega)}{\mathrm{d} t}=L\left(\theta_{t} \omega\right) \mathbf{u}+f\left(\theta_{t} \omega, \mathbf{u}\right) .
$$


First, since $\alpha\left(\theta_{t} \omega\right)$ is bounded, the operator $L$ generates an evolution system on $\mathbb{R}^{3}$. Second, since $\Lambda\left(\theta_{t} \omega\right)$ is continuous with respect to $t$, function $f$ is continuous with respect to $t$ and locally Lipschitz with respect to $\mathbf{u}$. Hence system (11) has a unique local solution $\mathbf{u}\left(\cdot ; t_{0}, \omega, \mathbf{u}_{0}\right) \in \mathscr{C}\left(\left[t_{0}, T\right), \mathbb{R}^{3}\right)$.

By continuity of solutions, each solution has to take value 0 before it reaches a negative value. Notice that

$$
\begin{aligned}
& \left.\frac{\mathrm{d} x(t, \omega)}{\mathrm{d} t}\right|_{x=0, y \geq 0, v \geq 0}=\Lambda\left(\theta_{t} \omega\right)>0, \\
& \left.\frac{\mathrm{d} y(t, \omega)}{\mathrm{d} t}\right|_{x \geq 0, y=0, v \geq 0}=\beta x v \geq 0, \\
& \left.\frac{\mathrm{d} v(t, \omega)}{\mathrm{d} t}\right|_{x \geq 0, y \geq 0, v=0}=\kappa y \geq 0,
\end{aligned}
$$

we have $x(t)$ strictly increasing at $x=0, y(t)$ and $v(t)$ non-decreasing at $y=0$ and $v=0$, respectively. This implies that $\mathbf{u}(t) \in \mathbb{R}_{+}^{3}$ for $t \in\left[t_{0}, T\right)$.

For $\mathbf{u}(t) \in \mathbb{R}_{+}^{3}$, define

$$
\|\mathbf{u}(t)\|_{1}:=x(t)+y(t)+v(t) .
$$

Let $s(t)=2 \kappa x(t)+2 \kappa y(t)+\gamma_{2} v(t)$, then

$$
\|\mathbf{u}(t)\|_{1} \leq \frac{s(t)}{\min \left\{2 \kappa, \gamma_{2}\right\}} .
$$

On the other hand by (6) - (8) we have

$$
\begin{aligned}
\frac{\mathrm{d} s(t, \omega)}{\mathrm{d} t} & =2 \kappa \Lambda\left(\theta_{t} \omega\right)-2 \kappa \gamma_{1} x-\kappa \gamma_{2} y-\gamma_{2} \alpha\left(\theta_{t} \omega\right) v \\
& \leq 2 \kappa \lambda\left(1+\delta_{1}\right)-2 \kappa \gamma_{1} x-\kappa \gamma_{2} y-\gamma_{2} a\left(1-\delta_{2}\right) v \\
& \leq 2 \kappa \lambda\left(1+\delta_{1}\right)-\mu_{1} s(t),
\end{aligned}
$$

where

$$
\mu_{1}=\min \left\{\gamma_{1}, \gamma_{2} / 2, a\left(1-\delta_{2}\right)\right\}>0 .
$$

For $s\left(t_{0}\right) \geq 2 \kappa \lambda\left(1+\delta_{1}\right) / \mu_{1}, s(t)$ will be non-increasing for $t \geq t_{0}$ and thus $s(t) \leq$ $s\left(t_{0}\right)$. Otherwise, for $s\left(t_{0}\right) \leq 2 \kappa \lambda\left(1+\delta_{1}\right) / \mu_{1}, s(t)$ will stay $\leq 2 \kappa \lambda\left(1+\delta_{1}\right) / \mu_{1}$. In summary,

$$
0 \leq\|\mathbf{u}\|_{1} \leq \frac{s(t)}{\min \left\{2 \kappa, \gamma_{2}\right\}} \leq \frac{\max \left\{2 \kappa x\left(t_{0}\right)+2 \kappa y\left(t_{0}\right)+\gamma_{2} v\left(t_{0}\right), 2 \kappa \lambda\left(1+\delta_{1}\right) / \mu_{1}\right\}}{\mu_{2}},
$$

where

$$
\mu_{2}=\min \left\{2 \kappa, \gamma_{2}\right\}
$$


This implies that system (11) has a unique positive and bounded global solution $\mathbf{u}\left(\cdot ; t_{0}, \omega, \mathbf{u}_{0}\right) \in \mathbb{R}_{+}^{3}$.

It is straightforward to check that

$$
\mathbf{u}\left(t+t_{0} ; t_{0}, \omega, \mathbf{u}_{0}\right)=\mathbf{u}\left(t ; 0, \theta_{t_{0}} \omega, \mathbf{u}_{0}\right)
$$

for all $t_{0} \in \mathbb{R}, t \geq t_{0}, \omega \in \Omega$ and $\mathbf{u}_{0} \in \mathbb{R}_{+}^{3}$. This allows us to define a mapping $\varphi(t, \omega)(\cdot)$ :

$$
\varphi(t, \omega) \mathbf{u}_{0}=\mathbf{u}\left(t ; 0, \omega, \mathbf{u}_{0}\right), \quad \forall t \geq 0, \mathbf{u}_{0} \in \mathbb{R}_{+}^{3}, \omega \in \Omega .
$$

From now on, we will simply write $\mathbf{u}\left(t ; \omega, \mathbf{u}_{0}\right)$ instead of $\mathbf{u}\left(t ; 0, \omega, \mathbf{u}_{0}\right)$.

For any $\mathbf{u}_{0} \in \mathbb{R}_{+}^{3}$, solution $\mathbf{u}\left(\cdot ; \omega, \mathbf{u}_{0}\right) \in \mathbb{R}_{+}^{3}$ for $t \in[0, \infty)$. Since function $f\left(\mathbf{u}, \theta_{t} \omega\right)=$ $f(\mathbf{u}, t, \omega)$ is continuous in $\mathbf{u}, t$, and is measurable in $\omega, \mathbf{u}:[0, \infty) \times \Omega \times \mathbb{R}_{+}^{3} \rightarrow$ $\mathbb{R}_{+}^{3},\left(t ; \omega, \mathbf{u}_{0}\right) \mapsto \mathbf{u}\left(t ; \omega, \mathbf{u}_{0}\right)$ is $\left(\mathscr{B}[0, \infty) \times \mathscr{F}_{0} \times \mathscr{B}\left(\mathbb{R}_{+}^{3}\right), \mathscr{B}\left(\mathbb{R}_{+}^{3}\right)\right)$-measurable. It then follows directly that (11) generates a continuous random dynamical system $\varphi(t, \omega)(\cdot)$ defined by $(15)$. This completes the proof.

\section{Existence and geometric structure of global random attractors}

In this section we will first prove the existence of a global random attractor for the random dynamical system $\{\varphi(t, \omega)\}_{t \geq 0, \omega \in \Omega}$. In addition, we will investigate the geometric structure of this random attractor.

Theorem 2. The random dynamical system generated by system (11) possesses a unique global random attractor $\mathscr{A}=\{A(\omega): \omega \in \Omega\}$.

Proof. We first prove that for $\omega \in \Omega$, there exists a tempered bounded closed random absorbing set $K(\omega) \in \mathscr{D}\left(\mathbb{R}_{+}^{3}\right)$ of the random dynamical system $\{\varphi(t, \omega)\}_{t \geq 0, \omega \in \Omega}$ such that for any $B \in \mathscr{D}\left(\mathbb{R}_{+}^{3}\right)$ and each $\omega \in \Omega$, there exists $T_{B}(\omega)>0$ yielding

$$
\varphi\left(t, \theta_{-t} \omega\right) B\left(\theta_{-t} \omega\right) \subset K(\omega) \quad \forall t \geq T_{B}(\omega) .
$$

In fact, recall that $\mathbf{u}\left(t ; \omega, \mathbf{u}_{0}\right)=\varphi(t, \omega) \mathbf{u}_{0}$ denotes the solution of system (11) satisfying $\mathbf{u}\left(0 ; \omega, \mathbf{u}_{0}\right)=\mathbf{u}_{0}$. Then for any $\mathbf{u}_{0}:=\mathbf{u}_{0}\left(\theta_{-t} \omega\right) \in B\left(\theta_{-t} \omega\right)$,

$$
\left\|\varphi\left(t, \theta_{-t} \omega\right) \mathbf{u}_{0}\right\|_{1}=\left\|\mathbf{u}\left(t ; \theta_{-t} \omega, \mathbf{u}_{0}\left(\theta_{-t} \omega\right)\right)\right\|_{1} \leq \frac{1}{\mu_{2}} \cdot s\left(t ; \theta_{-t} \omega, s_{0}\left(\theta_{-t} \omega\right)\right) .
$$

Using inequality (12) and substituting $\omega$ by $\theta_{-t} \omega$ we obtain

$$
\begin{aligned}
s\left(t ; \theta_{-t} \omega, s_{0}\left(\theta_{-t} \omega\right)\right) s_{0} & \leq e^{-\mu_{1} t}+\frac{2 \kappa \lambda\left(1+\delta_{1}\right)}{\mu_{1}} \\
& \leq e^{-\mu_{1} t} \sup _{(x, y, v) \in B\left(\theta_{-t} \omega\right)}\left(2 \kappa x+2 \kappa y+\gamma_{2} v\right)+\frac{2 \kappa \lambda\left(1+\delta_{1}\right)}{\mu_{1}} .
\end{aligned}
$$


Therefore for any $\varepsilon>0$, and $\mathbf{u}_{0} \in B\left(\theta_{-t} \omega\right)$, there exists $T_{B}(\omega)$ such that when $t>T_{B}$,

$$
\begin{aligned}
\left\|\varphi\left(t, \theta_{-t} \omega\right) \mathbf{u}_{0}\right\|_{1} & \leq \frac{1}{\mu_{2}} \cdot s\left(t ; \theta_{-t} \omega, s_{0}\left(\theta_{-t} \omega\right)\right) \\
& \leq \frac{1}{\mu_{2}} \cdot \frac{2 \kappa \lambda\left(1+\delta_{1}\right)}{\mu_{1}}+\varepsilon
\end{aligned}
$$

Define

$$
K_{\varepsilon}(\omega)=\overline{\left\{(x, y, v) \in \mathbb{R}_{+}^{3}: x+y+v \leq \frac{1}{\mu_{2}} \cdot \frac{2 \kappa \lambda\left(1+\delta_{1}\right)}{\mu_{1}}+\varepsilon\right\}} .
$$

Then $K_{\varepsilon}(\omega)$ is positively invariant and absorbing in $\mathbb{R}_{+}^{3}$.

It follows directly from Proposition 1, that the random dynamical system generated by system (6) - (8) possesses a random attractor $\mathscr{A}=\{A(\omega): \omega \in \Omega\}$, consisting of nonempty compact random subsets of $\mathbb{R}_{+}^{3}$ contained in $K_{\mathcal{\varepsilon}}(\omega)$. This completes the proof.

Next we will investigate details of the random attractor $\mathscr{A}$.

Theorem 3. The random pullback attractor $\mathscr{A}=\{A(\omega): \omega \in \Omega\}$ for the random dynamical system generated by system (6) - (8) has singleton component sets $A(\omega)$ $=\left\{\left(x^{*}(\omega), 0,0\right)\right\}$ for every $\omega \in \Omega$, provided that

$$
\frac{\kappa}{\gamma_{2}} \leq 1 \quad \text { and } \quad \frac{\beta \lambda\left(1+\delta_{1}\right)}{\mu_{1} a\left(1-\delta_{2}\right)}<1
$$

Proof. Sum (7) and (8) we obtain

$$
\frac{\mathrm{d}(y+v)}{\mathrm{d} t}=-\left(\gamma_{2}-\kappa\right) y-\left(\alpha\left(\theta_{t} \omega\right)-\beta x\right) v .
$$

Recall that due to (16), for any $\varepsilon>0$, there exists $T_{B}(\omega)$ such that when $t>T_{B}$,

$$
x(t) \leq\|\mathbf{u}(t)\|_{1} \leq \frac{1}{\mu_{2}} \cdot \frac{2 \kappa \lambda\left(1+\delta_{1}\right)}{\mu_{1}}+\varepsilon .
$$

By definition of $\mu_{2}$ in (14), we have that $2 \kappa / \mu_{2} \leq 1$. Then pick $\varepsilon$ small enough we have

$$
\begin{aligned}
\alpha\left(\theta_{t} \omega\right)-\beta x & >\alpha\left(1-\delta_{2}\right)-\beta \cdot \frac{1}{\mu_{2}} \cdot \frac{2 \kappa \lambda\left(1+\delta_{1}\right)}{\mu_{1}} \\
& \geq \alpha\left(1-\delta_{2}\right)-\beta \cdot \frac{\lambda\left(1+\delta_{1}\right)}{\mu_{1}}>0,
\end{aligned}
$$

which implies that $y+v$ decreases to 0 as $t$ approaches $\infty$.

Letting $y=v=0$ in equation (6), we obtain 


$$
\frac{\mathrm{d} x}{\mathrm{~d} t}=\Lambda\left(\theta_{t} \omega\right)-\gamma_{1} x
$$

Solving equation (18) gives

$$
x\left(t ; \omega, x_{0}\right)=x_{0} e^{-\gamma_{1} t}+\int_{0}^{t} \Lambda\left(\theta_{s} \omega\right) e^{\gamma_{1}(t-s)} \mathrm{d} s,
$$

and consequently

$$
x\left(t ; \theta_{-t} \omega, x_{0}\right)=x_{0} e^{-\gamma_{1} t}+\int_{-t}^{0} \Lambda\left(\theta_{s} \omega\right) e^{-\gamma_{1} s} \mathrm{~d} s \stackrel{t \rightarrow \infty}{\longrightarrow} \int_{-\infty}^{0} \Lambda\left(\theta_{s} \omega\right) e^{-\gamma_{1} s} \mathrm{~d} s:=x^{*}(\omega) .
$$

This completes the proof.

Theorem 3 implies that $\left(x^{*}\left(\theta_{t} \omega\right), 0,0\right)$ is asymptotically stable as $t \rightarrow \infty$, i.e., endemic occurs when the parameters satisfy (17). We next investigate the conditions under which epidemic occurs.

Theorem 4. The random pullback attractor $\mathscr{A}=\{A(\omega): \omega \in \Omega\}$ for the random dynamical system generated by system (6) - (8) possesses nontrivial component sets which include $\left(x^{*}(\omega), 0,0\right)$ and strictly positive points provided that

$$
\frac{\beta \lambda\left(1+\delta_{1}\right)}{\mu_{1} a\left(1+\delta_{2}\right)}>\frac{\gamma_{2}}{\kappa} .
$$

Proof. First notice that the equation (7) is deterministic, and implies that the surface $y=\frac{\beta}{22} x v$ is invariant. The dynamics of $x$ and $v$ restricted on this invariant surface
satisfy

$$
\begin{aligned}
& \frac{\mathrm{d} x(t, \omega)}{\mathrm{d} t}=\Lambda\left(\theta_{t} \omega\right)-\gamma_{1} x-\beta x v \\
& \frac{\mathrm{d} v(t, \omega)}{\mathrm{d} t}=\frac{\kappa \beta}{\gamma_{2}} x v-\alpha\left(\theta_{t} \omega\right) v .
\end{aligned}
$$

Define the region $\Gamma_{\varepsilon}$ by

$\Gamma_{\varepsilon}:=\left\{(x, v) \in \mathbb{R}_{+}^{2}: x \geq \frac{a\left(1+\delta_{2}\right) \gamma_{2}}{\kappa \beta}+\varepsilon, v \geq \varepsilon, \frac{\kappa}{\gamma_{2}} x(t)+v(t) \leq \frac{\kappa \lambda}{\mu_{1} \gamma_{2}}\left(1+\delta_{1}\right)+\varepsilon\right\}$.

For any $(x, v) \in \Gamma_{\varepsilon}$ we have

$$
\frac{\mathrm{d} v}{\mathrm{~d} t}=\left(\frac{\kappa \beta}{\gamma_{2}} x-\alpha\left(\theta_{t} \omega\right)\right) v>\left(\frac{\kappa \beta}{\gamma_{2}} \cdot \frac{a\left(1+\delta_{2}\right) \gamma_{2}}{\kappa \beta}-a\left(1+\delta_{2}\right)\right) v \geq 0 .
$$

On the other hand, we have 


$$
\begin{aligned}
\frac{\mathrm{d}}{\mathrm{d} t}\left(\frac{\kappa}{\gamma_{2}} x(t)+v(t)\right) & =\frac{\kappa}{\gamma_{2}} \Lambda\left(\theta_{t} \omega\right)-\gamma_{1} \frac{\kappa}{\gamma_{2}} x-\alpha\left(\theta_{t} \omega\right) v \\
& \leq \frac{\kappa \lambda}{\gamma_{2}}\left(1+\delta_{1}\right)-\gamma_{1} \frac{\kappa}{\gamma_{2}} x-a\left(1-\delta_{2}\right) v \\
& \leq \frac{\kappa \lambda}{\gamma_{2}}\left(1+\delta_{1}\right)-\mu_{1}\left(\frac{\kappa}{\gamma_{2}} x(t)+v(t)\right),
\end{aligned}
$$

where $\mu_{1}$ is as defined in (13). This implies that

$$
\frac{\kappa}{\gamma_{2}} x(t)+v(t) \leq \frac{\kappa \lambda}{\mu_{1} \gamma_{2}}\left(1+\delta_{1}\right)+\varepsilon
$$

for $t$ large enough. Assumption (19) ensures that $\Gamma_{\varepsilon}$ is a nonempty compact positive invariant absorbing set, which then ensures the existence of a nontrivial pullback attractor $\mathscr{A}_{\mathcal{E}}=\left\{A_{\mathcal{E}}(t): t \in \mathbb{R}\right\}$ in $\Gamma_{\mathcal{\varepsilon}}$. This completes the proof.

\section{Numerical simulations}

In this section, we will simulate the system (6) - (8) numerically and verify that conditions (17) and (19) give rise to an endemic state (all infected cells and viruses are cleared) and a pandemic state (susceptible cells, infected cells, and viruses coexist) of system (6) - (8), respectively.

First we transform the system (6) - (8) and two OU processes $Z_{1}(t), Z_{2}(t)$ into a system of random ordinary differential equation (RODE) - stochastic ordinary differential equation (SODE) pair [2, 4]:

$$
d\left(\begin{array}{c}
x(t) \\
y(t) \\
v(t) \\
Z_{1}(t) \\
Z_{2}(t)
\end{array}\right)=\left(\begin{array}{c}
\Lambda\left(Z_{1}\right)-\gamma_{1} x-\beta x v \\
\beta x v-\gamma_{2} y \\
\kappa y-\alpha\left(Z_{2}\right) \\
\theta_{11}-\theta_{12} Z_{1} \\
\theta_{21}-\theta_{22} Z_{2}
\end{array}\right) d t+\left(\begin{array}{c}
0 \\
0 \\
0 \\
\theta_{13} \\
\theta_{23}
\end{array}\right) d W_{t}
$$

The OU processes $Z_{1}(t)$ and $Z_{2}(t)$ can be generated independently and we solve only the RODE part, i.e., $x, y$ and $v$ compartments, of the RODE-SODE system. The system is assumed to be stiff and the implicit 1.5-order RODE-Taylor scheme in [2] is applied here.

In the following simulation, we suppose that the cell proliferation rate $\Lambda\left(Z_{1}\right)$ has a switching effect and the loss rate of viruses $\alpha\left(Z_{2}\right)$ is distributed in a finite interval. They are randomized by the equations (9) and (10), respectively and given by 


$$
\begin{aligned}
& \Lambda\left(Z_{1}\right)=\lambda\left(1-2 \delta_{1} \frac{Z_{1}}{1+Z_{1}^{2}}\right), \\
& \alpha\left(Z_{2}\right)=a\left(1-\frac{2 \delta_{2}}{\pi} \arctan Z_{2}\right),
\end{aligned}
$$

which satisfy (4) and (5).

Initial conditions for $x, y$ and $v$ compartments are set as $x_{0}=2 \times 10^{5}, y_{0}=1 \times 10^{5}$ and $v_{0}=1 \times 10^{6}$. The coefficients for the OU processes are fixed to $\theta_{11}=1, \theta_{12}$ $=3, \theta_{13}=0.8, \theta_{21}=0, \theta_{22}=1$ and $\theta_{23}=0.5$ for all examples. We will choose different set of parameters that satisfy assumption (17) or assumption (19).

\section{Example 1}

In this example we set the parameters to be $\gamma_{1}=0.25, \gamma_{2}=0.5, \beta=1 \times 10^{-5}, \lambda=$ $4 \times 10^{4}, a=3, \delta_{1}=0.45, \delta_{2}=0.2$, and $\kappa=0.2$. Assumptions (17) is satisfied by this set of parameters. Figure 1 shows that the $y$ and $v$ compartments go to zero after enough amount of time and only $x$ compartment remains non zero, which means that the endemic state is achieved for parameters satisfying (17).

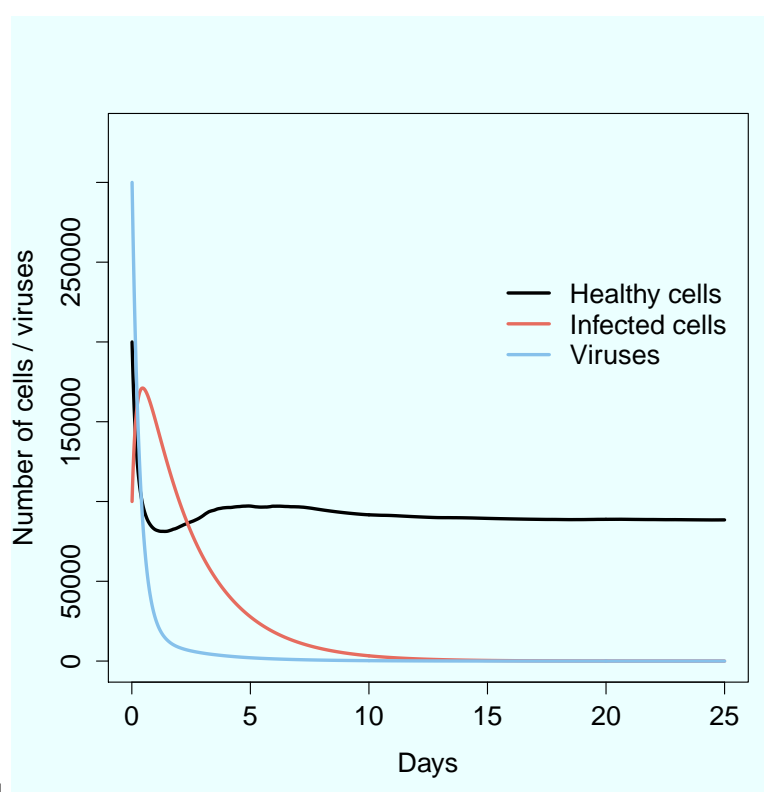

[ht]

Fig. 1 With parameters $\gamma_{1}=0.25, \gamma_{2}=0.5, \beta=1 \times 10^{-5}, \lambda=4 \times 10^{4}, a=3, \delta_{1}=0.45, \delta_{2}=$ 0.2 , and $\kappa=0.2$ satisfying assumption (17), both infect cells and viruses are cleared; only healthy cells remain. 


\section{Example 2}

In this example we set the parameters to be $\gamma_{1}=0.25, \gamma_{2}=0.5, \beta=1 \times 10^{-5}, \lambda=$ $4 \times 10^{4}, a=3, \delta_{1}=0.45, \delta_{2}=0.2$, and $\kappa=2$. Assumptions (19) is satisfied by this set of parameters. Figure 2 shows that $x, y$ and $v$ all remain non zero for a time long enough, which means that the pandemic state is achieved for parameters satisfying (19).

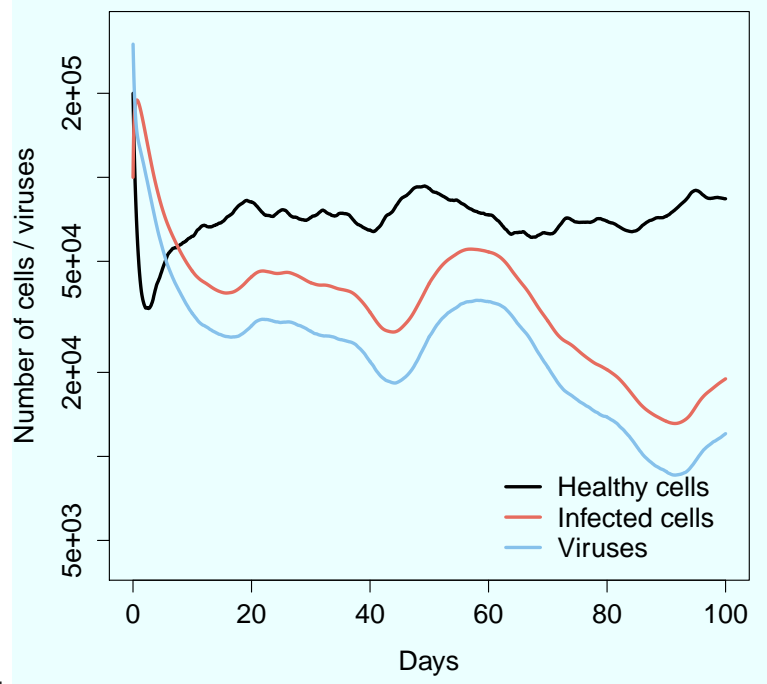

[ht]

Fig. 2 With parameters $\gamma_{1}=0.25, \gamma_{2}=0.5, \beta=1 \times 10^{-5}, \lambda=4 \times 10^{4}, a=3, \delta_{1}=0.45, \delta_{2}=$ 0.2 , and $\kappa=2$ satisfying assumption (19), infected cells, susceptible cells and viruses coexist.

Notice that the only parameter that has different values in Example 1 and Example 2 is $\kappa$. This implies that the rate at which virus is generated by dead susceptible cells is critical. A series of numerical simulations with different parameters were done to support this argument, among which we picked one more example to present below. In the following example, the parameters are chosen to be $\gamma_{1}=0.4, \gamma_{2}=0.5$, $\beta=5 \times 10^{-5}, \lambda=10^{5}, a=5, \delta_{1}=0.4, \delta_{2}=0.2$. When $\kappa=0.3$, assumption (17) is satisfied and we obtain an endemic state (see Figure 3). When $\kappa=3$, assumption (19) is satisfied and we obtain a pandemic state (see Figure 4).

Acknowledgements This work has been partially supported by the Chinese NSF grant no. 1157112, the Spanish Ministerio de Economía y Competitividad project MTM2015-63723-P and the Consejería de Innovación, Ciencia y Empresa (Junta de Andalucía) under grant 2010/FQM314 and Proyecto de Excelencia P12-FQM-1492. 


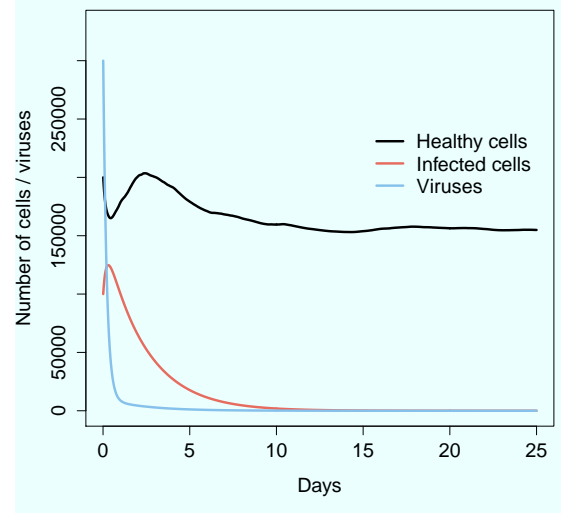

Fig. 3 With parameters $\gamma_{1}=0.4, \gamma_{2}=0.5, \beta=$ $5 \times 10^{-5}, \lambda=10^{5}, a=5, \delta_{1}=0.4, \delta_{2}=0.2$ and $\kappa=0.3$ satisfying assumption (17), both infect cells and viruses are cleared; only healthy cells remain.

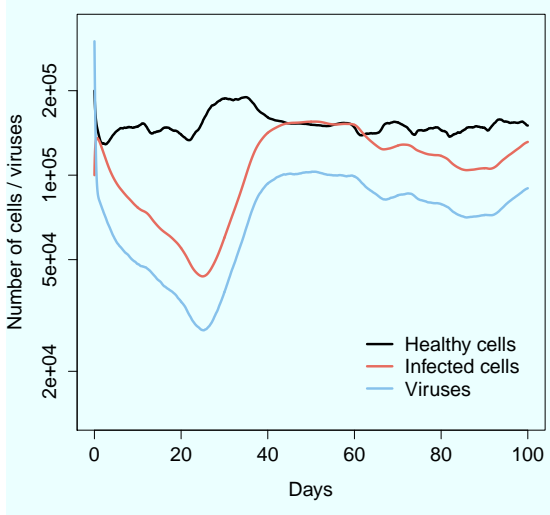

Fig. 4 With parameters $\gamma_{1}=0.4, \gamma_{2}=0.5, \beta=$ $5 \times 10^{-5}, \lambda=10^{5}, a=5, \delta_{1}=0.4, \delta_{2}=0.2$ and $\kappa=3$ satisfying assumption (19), infected cells, susceptible cells and viruses coexist.

\section{References}

1. L. Arnold, Random Dynamical Systems, Springer-Verlag, Berlin (1998).

2. Y. Asai and P.E. Kloeden, Numerical schemes for random ODEs via stochastic differential equations, Commun. Appl. Analysis 17 (2013), 521-528.

3. Y. Asai, E. Herrmann and P.E. Kloeden, Stable integration of stiff random ordinary differential equations, J. Stoch. Anal. Applns. 31 (2013) 293-313.

4. Y. Asai and P.E. Kloeden, Multi-step methods for random ODEs driven by Itô diffusions, $J$. Comput. Appl. Math 294 (2016), 210-224.

5. P. W. Bates, H. Lisei and K. Lu, Attractors for stochastic lattice dynamical systems, Stochastics and Dynamics 6 (2006), 1-21.

6. T. Caraballo, X. Han and P. E. Kloeden, Chemostats with random inputs and wall growth, Math. Meth. Appl. Sci. (2015) doi: 10.1002/mma.3437.

7. T. Caraballo, X. Han and P. E. Kloeden, Nonautonomous chemostats with varible delays, SIAM J. Math. Anal. 47 (2015) 2178 - 2199.

8. T. Caraballo, P.E. Kloeden and B. Schmalfuss, Exponentially stable stationary solutions for stochastic evolution equations and their perturbation. Appl. Math. Optim., 50 (2004), 183-207.

9. T. Caraballo, G. Lukaszewicz and J. Real, Pullback attractors for asymptotically compact nonautonomous dynamical systems, Nonlinear Analysis TMA 6 (2006), 484-498.

10. F. Flandoli and B. Schmalfuss, Random attractors for the 3D stochastic Navier-Stokes equation with multiplicative noise, Stochastics Stochastics Rep. 59 (1996), no. 1-2, 21-45.

11. P.E. Kloeden and T. Lorenz, Pullback incremental attraction, Nonautonomous \& Random Dynamical Systems (2013), 53-60 DOI: 10.2478/msds-2013-0004

12. R. May and M. Nowak, Virus Dynamics: Mathematical Principles of Immunology and Virology, Oxford University Press, Oxford (2001).

13. A.S. Perelson and R.M. Ribeiro, Modeling the within-host dynamics of HIV infection, $B M C$ Biology 11 (2013) 96. 